\title{
A Comparison of the Accuracy of WATCHMAN and WATCHMAN FLX Device Sizing between CT, TEE, and Patient Specific 3D Models
}

\author{
Ivette Troitino' ${ }^{1}$, T. Eric White', John Lozo ${ }^{3}$ \\ ${ }^{1}$ Indiana University School of Medicine, ${ }^{2}$ Parkview Physicians Group- Cardiology, ${ }^{3}$ Parkview \\ Mirro Center for Research and Innovation
}

Background and Hypothesis: In patients with Atrial fibrillation (AF), the Left Atrial Appendage (LAA) is the most common site of thrombus formation. The LAA occlusion procedure using the WATCHMAN device implant is an alternative for stroke prevention in AF patients. Transesophageal echocardiogram (TEE) and Computed tomography (CT) scans aid in measuring the LAA to predict implant device sizes. However, due to varying LAA anatomy and limited spatial resolution, the current imaging techniques often predict one of two sized devices. The objective of this retrospective study is to compare the accuracy of measurements made preoperatively of the LAA with those on 3D models to determine if they can be used in preoperative planning. We hypothesize 3D models will be more accurate in predicting device size and any anatomical impediments than traditional TEE planning.

Project Methods: There were 21 subjects selected who underwent the WATCHMAN FLX procedure at Parkview Heart Institute in 2021. 3D models of LAA were created from CT scans using a Form $23 \mathrm{D}$ printer. The device sizes predicted for the procedure were determined from Boston Scientific FLX guidelines based on the maximum LAA orifice diameter from TEE, CT, and 3D models.

Results: Two-proportion z-test between the 3D model predicted sizes to the actual size deployed demonstrated no statistical significance $(p=0.298)$ demonstrating no difference between 3D model predicted sizes and actual size deployed. Two-proportion z-test between TEE vs actual size and CT vs actual size demonstrated statistical significance, suggesting a difference between the group's predictions. 3D models predicted the accurate device size for $20 / 21(95 \%)$ subjects. TEE measurements of maximum orifice diameter were, on average, lower compared to CT and 3D model measurements.

Conclusion and Potential Impact: 3D printed models provide the most accurate device size predictions and can be used to optimize presurgical planning while reducing intraoperative complications. 